\title{
Improvement of Position Accuracy with GAGAN and the Impact of Scintillation on GNSS
}

\author{
Surendra Sunda ${ }^{1 *}$, B. M. Vyas ${ }^{2}$, S. V. Satish ${ }^{3}$, P. V. Khekale ${ }^{4}$, K. S. Parikh ${ }^{4}$ \\ ${ }^{1}$ Airports Authority of India, Ahmedabad, India; ${ }^{2}$ Department of Physics, M. L. S. University, Udaipur, India; ${ }^{3}$ Airports Authority of \\ India, New Delhi, India; ${ }^{4}$ Space Applications Centre, Ahmedabad, India. \\ Email: ssunda@gmail.com
}

Received August 22 ${ }^{\text {nd }}, 2013$; revised September 22 ${ }^{\text {nd }}, 2013$; accepted September $29^{\text {th }}, 2013$

Copyright (c) 2013 Surendra Sunda et al. This is an open access article distributed under the Creative Commons Attribution License, which permits unrestricted use, distribution, and reproduction in any medium, provided the original work is properly cited.

\begin{abstract}
GAGAN is an Indian implementation of Satellite Based Augmentation System (SBAS), developed jointly by Airports Authority of India (AAI) and Indian Space Research Organisation (ISRO). It is in final operational phase with all the required ground and space segments ready. With the availability of GAGAN signal-in-space (SIS), the improvement in position solution is investigated using the two collocated dual frequency GPS receivers. One of the receivers was configured as SBAS receiver and the other was kept as GPS stand-alone receiver. It is found that accuracy in position improved significantly in SBAS receiver due to GAGAN correction. The impact of scintillation on GNSS was also investigated in terms of position degradation and loss of lock of the satellite signals. The manyfold effects of scintillation on GPS and SBAS are discussed in detail. The results indicate $\sim 15 \%$ reduction in number of measurements due to loss of lock during severe scintillation.
\end{abstract}

Keywords: GAGAN; SBAS; Position; Scintillation; GPS

\section{Introduction}

GPS Aided Geo Augmented Navigation (GAGAN) is a joint project of Airports Authority of India and Indian Space Research Organisation (ISRO) to provide the seamless navigation to civil aviation users. It is the Indian implementation of Satellite Based Augmentation System (SBAS), similar to US-WAAS, Europe's EGNOS and Japanese MSAS. The objective of SBAS implementation is to improve the accuracy, integrity, availability and continuity of navigation services in all phases of flight i.e. from en-route to approach. After successful completion of Technology Demonstration System (TDS) in 2007, GAGAN entered into Final Operational Phase (FOP). The ground segment of GAGAN, at present, comprises of 15 Indian Reference Stations (INRES), 3 Indian Land Uplink Stations (INLUS), 2 Indian Master Control Centres (INMCC) and the redundant communication network to interconnect these segments. The space segment consisted of GPS satellites and two Geostationary Earth Orbiting (GEO) satellites: 1) GSAT-8 launched in May, 2011 and located at $55^{\circ} \mathrm{E}$, transmitting the PRN code-

*Attached to Space Applications Centre.
127 and 2) GSAT-10 launched in September, 2012 and located at $83^{\circ} \mathrm{E}$, transmitting the PRN code- 128 . Both the GEO satellites have been integrated with uplink stations to transmit the GAGAN correction and integrity messages.

Apart from the above configuration, a network of dual frequency GPS receivers outputting ionospheric parameters-Total Electron Content (TEC) and scintillation measurements $\left(\mathrm{S}_{4}\right)$ was established in 2003-2004, referred as GAGAN-TEC network to study and characterise the ionosphere for development of grid based iono model [1]. In total, 18 receivers were installed under GAGAN-TEC network at various airports which were increased to 26 in 2009-2010. These dual frequency GPS (TEC) receivers are also capable of logging SBAS correction messages. In addition to that, they can be configured to provide the position solution by applying the SBAS (GAGAN) corrections. This feature can be efficiently utilised for analysing the performance of GAGAN in navigation/position solution. The location of TEC receivers can be used as user test position to evaluate the performance of GAGAN in terms of position accuracy. Additionally, the integrity parameters-Horizontal and Vertical Protection 
Limits (HPL/VPL) can also be calculated at these user test points [2].

This paper presents the preliminary analysis carried out to investigate the improvement in position accuracy with the help of GAGAN correction messages. Furthermore, a detailed study was carried out to investigate the effects of scintillation on satellite navigation in terms of position degradation and loss of lock.

\section{Experimental Setup}

Since the GAGAN signal-in-space (SIS) is available providing the range error corrections, it is pertinent to study the extent of improvement it provides in position solution. For this purpose, an experiment was carried out at Ahmedabad $\left(23.02^{\circ} \mathrm{N}, 72.51^{\circ} \mathrm{E}\right)$ using two collocated dual frequency GPS receivers "GSV4004B". One receiver was configured to track and acquire the SBAS (GEO) satellites: GSAT-8 and GSAT-10. Furthermore, it was configured to provide the position solution using GAGAN corrections i.e. to act as SBAS receiver. Since GAGAN is, currently, transmitting test message MT-0 (do not use) [2], the receiver was configured to ignore this message to apply the GAGAN corrections in computing the position solution. Thereafter, the position information was logged at one minute interval for two months. Similarly, the position information was logged in the other receiver, which was kept as simple GPS stand-alone receiver. Since the exact surveyed positions of these receiver antennas was not known, we averaged the "Bestpos" information over a period of one month to find the standard position of the antennas.

\section{Results of Experiment}

The position error is calculated at each minute by subtracting the computed position from the standard position. Figure 1 shows the scatter plot of the position error in SBAS and GPS stand-alone receiver for 24-hour duration on a typical day of 11 March, 2013. It can be noticed that the spread of SBAS error is very less as compared to that of GPS stand-alone. The longitude error spread is limited to $\sim 2$ meters, while it is $\sim 6$ meters for latitude error in SBAS receiver. Similarly, the longitude and latitude errors in GPS stand-alone are $\sim 8$ and 12 meters respectively implying the improvement in position accuracy using GAGAN corrections. Further, in order to see the improvement in Cartesian coordinates of $\mathrm{X}, \mathrm{Y}$, and $\mathrm{Z}$, we compared both receivers as shown in Figure 2. The upper panel in this Figure shows the position error (meter) for $\mathrm{X}, \mathrm{Y}$, and $\mathrm{Z}$ coordinates for SBAS receiver while the lower panel is for GPS stand-alone receiver for the same day of 17 February 2013. It can be noticed that, in general, error is more in Y coordinate for both the receivers. The maximum error in SBAS receiver is less than 4 me-

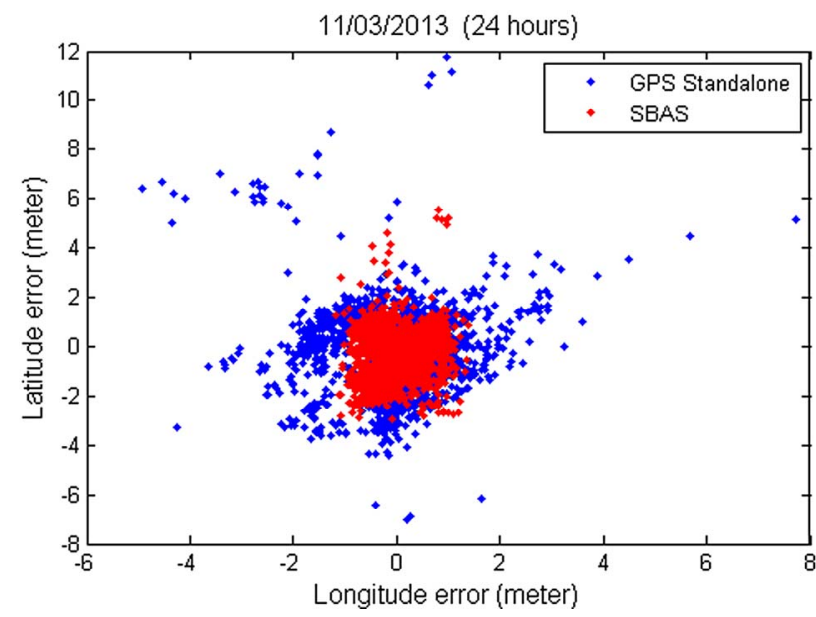

Figure 1. Scatter plot of position error in SBAS and GPS stand-alone receiver for 24 hours on 11 March, 2013.
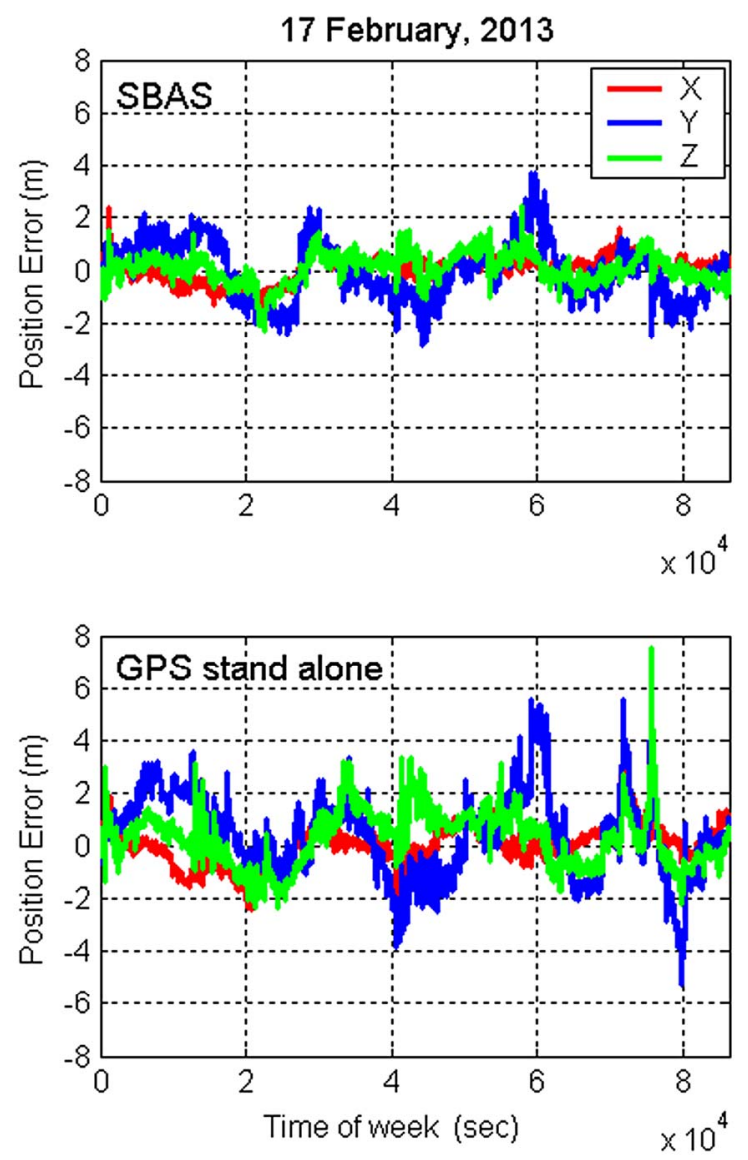

Figure 2. Comparison of position error in Cartesian coordinates for SBAS and GPS stand alone receivers on 17 February, 2013.

ter during 24 hours, whereas it is $~ 8$ meters in GPS stand alone receiver. This again shows that the position accuracy has been improved by applying the GAGAN correction messages.

To investigate the consistency of the results, we ana- 
lysed the position error for about 2 months. For this, we derived the 3-d rms error i.e.

$\sqrt{\left(\left(\sum\left(\delta X^{2}+\delta Y^{2}+\delta Z^{2}\right)\right) / n\right)}$ for each day, where $n$ is the number of epochs per day. This provides the dayto-day variation of 3-d rms error as shown in Figure 3 for SBAS and GPS stand alone receivers. The 3-d rms error, in SBAS, lies in the range of 1 - 1.5 meters with little variation, whereas, for GPS stand-alone receiver, significant variations are noticed within the range of 2 - 3 meters. The mean of 3-d rms error over 2 months for SBAS is $1.36 \mathrm{~m}$ with its standard deviation of just 0.09 , while for GPS stand-alone it is $2.47 \mathrm{~m}$ with standard deviation of 0.25 .

This experiment clearly indicates that GAGAN makes a significant improvement in position solution and it also achieves the required accuracy of 7.6 meters for APV1.5 navigation services [3]. The noticeable improvement in position accuracy with GAGAN is all the more significant since the test receiver "GPS stand-alone" is dual frequency receiver, which itself can take care of ionospheric errors. Hence the improvement observed may be largely attributed to orbital and clock corrections transmitted in GAGAN SIS.

\section{Impact of Scintillation on GNSS}

The scintillations are defined as small-scale irregularities in the ionosphere resulting from plasma bubbles (depleted plasma) that form at the bottom of the F-region ionization layer and percolate upward through the topside ionosphere. These irregularities map down the magnetic field lines towards the higher latitudes depending upon the altitude of the topside ionosphere. The higher altitudes will help the plasma bubbles to reach the Equatorial Ionization Anomaly (EIA) region $\left( \pm 15^{\circ}\right.$ of magnetic equator) to produce strong irregularities because the background electron density is relatively higher as compared to that of the magnetic equator [4].

The impacts of scintillation on the performance of satellite navigation are manifold. The signal fluctuations

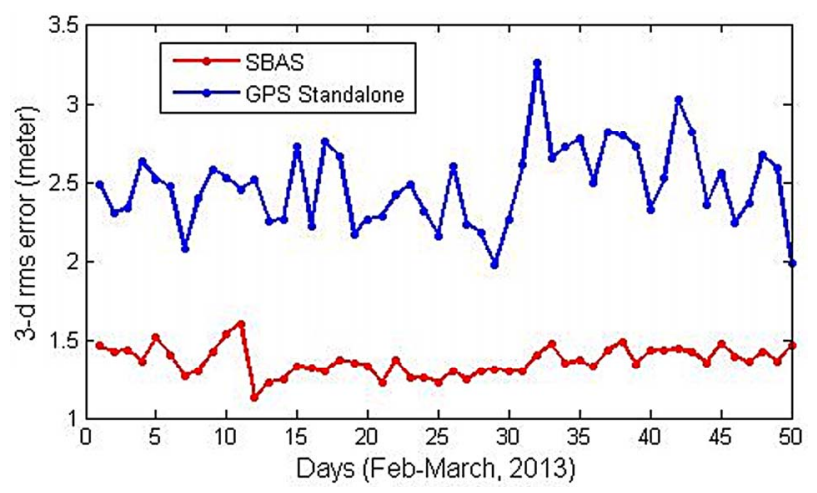

Figure 3. Day-to-day variation of 3-d rms position error over 2 months (February-March, 2013). during scintillation may reduce the accuracy of raw GPS measurements, thus increasing the positioning error. During intense scintillation, signal level drops below the threshold level of receiver's margin leading to loss of lock and cycle slips [5-8]. Simultaneous loss of two or more GPS satellites can severely degrade the accuracy of SBAS service and affect the availability due to undersampling of ionospheric pierce points. Severe scintillation over a large coverage area can potentially cause the short service outage [9]. Since SBAS comprises of ground segment (Reference stations, Master control centre and Uplink station), user segment (SBAS receivers at L1/L5), and space segment (GPS and geostationary satellites); all tied in a loop, the scintillation effect at any of the elements of these segments will manifest in the form of degraded accuracy and reduced availability of the service as shown by schematic diagram in Figure 4. We will investigate, in next section, how the severe scintillation causes the position degradation and further next, the quantitative effects of loss of lock.

\subsection{Scintillation Effects on Position}

In order to see the effect of scintillation on position, we relied on the experimental position data of about 2 months comprising SBAS and GPS stand-alone receivers as explained earlier. A very large 3-d rms error of $\sim 25$ meter in GPS stand-alone and $\sim 5$ meter in SBAS was noticed on same day (11 March, 2013) and around same time as shown in Figure 5(a). To investigate the cause of this error, we analyzed the scintillation measurements at Ahmedabad. As expected, the occurrence of very strong scintillation was observed on 11 March, 2013 as shown in Figure 5 (b). This figure shows the strength of amplitude scintillation " $\mathrm{S}_{4}$ " with time exhibiting severe scintillation $\left(\mathrm{S}_{4}>0.7\right)$ after $\sim 1500$ UT. It can also be noticed from these figures that severe scintillations during $\sim 1500$ - 1600 UT coincides with the increased position error. This implies that scintillation had severely affected the position accuracy both in SBAS as well as in GPS stand-alone receiver. It is well established that the user position error is proportional to the Geometrical dilution of precision (GDOP), which depends on the geometry of visible satellites. During severe scintillation, due to loss of lock of the multiple satellites, GDOP may degrade potentially to affect the position accuracy. In the next section, we will discuss the various implications of the loss of lock on GPS and SBAS.

\subsection{Loss of Lock Due to Scintillation}

Continuing with the previous example of 11 March, 2013, we will first investigate the loss of lock of SBAS (GEO) satellites due to scintillation. As mentioned in introduction, there are two operational GEO satellites for GA- 


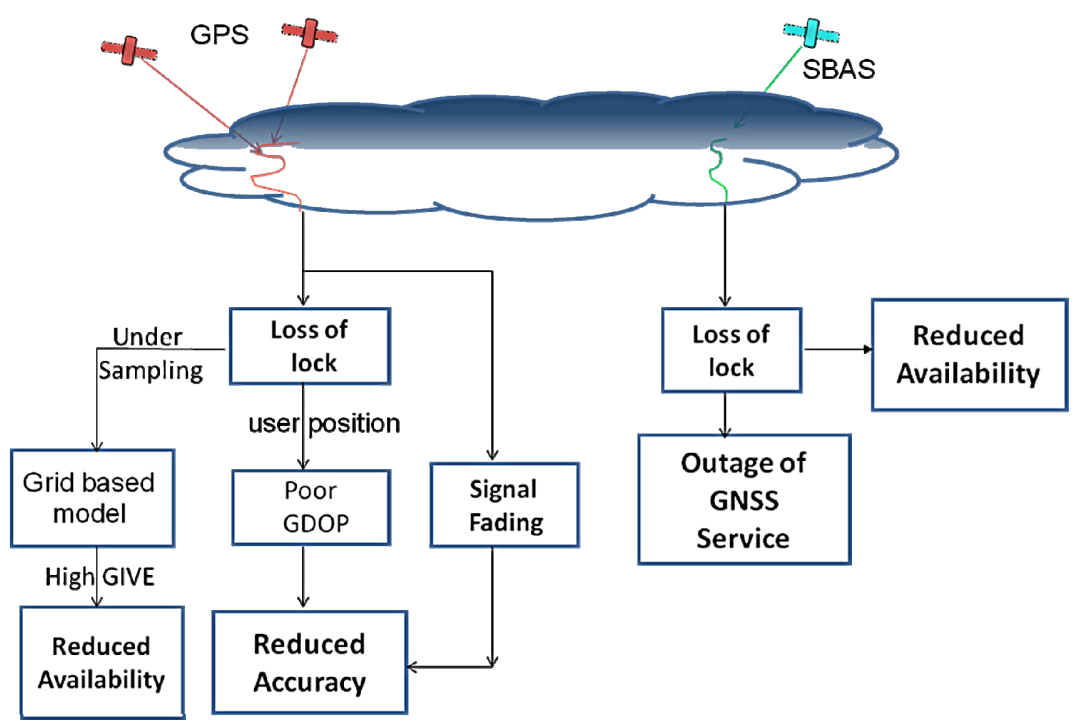

Figure 4. Schematic diagram showing the effects of scintillation on GNSS/SBAS.
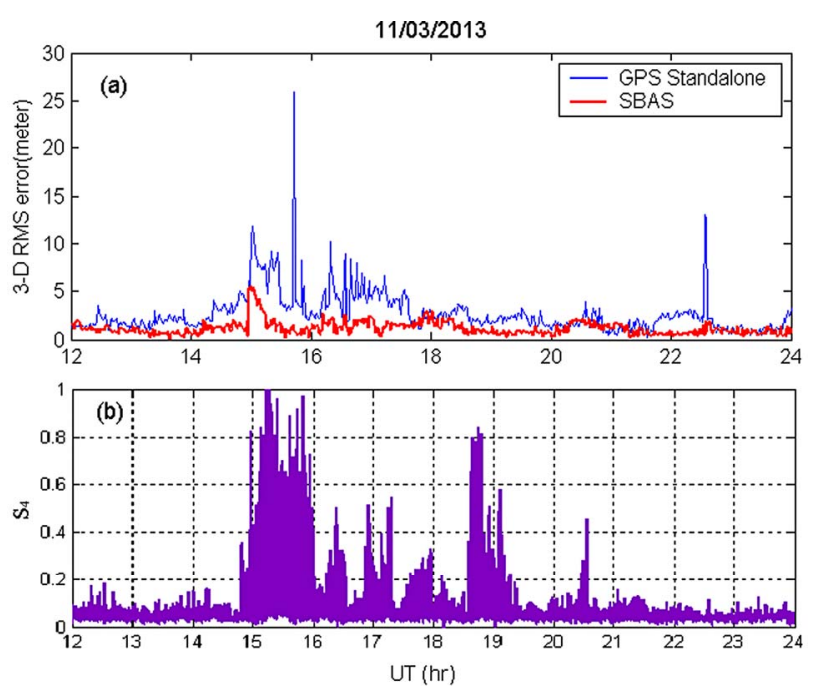

Figure 5. (a) Position error in SBAS and GPS stand-alone receiver on 11 March, 2013. (b) Amplitude scintillation $S_{4}$ on 11 March, 2013 at Ahmedabad.

GAN located at $55^{\circ} \mathrm{E}$ and $83^{\circ} \mathrm{E}$. The upper panel of Figure 6(a) shows the temporal variations of amplitude scintillation $\mathrm{S}_{4}$ observed on GSAT-8 (PRN 127) at Ahmedabad while the bottom panel shows the lock time of the satellite in seconds. The lock time is the incremental counter while satellite remained locked with the receiver, which gets reset to zero during loss of lock. It can be noticed that frequent loss of locks are observed in GSAT8 during severe scintillation period of $\sim 1500$ - 1600 UT. Surprisingly and alarmingly, severe scintillations leading to loss of lock are also observed on another GEO (GSAT10, PRN 128) as shown in Figure 6(b) implying large coverage area of scintillation. However, there is little bit offset in the occurrence time of scintillation between GSAT-8 and GSAT-10 due to spatial separation in their
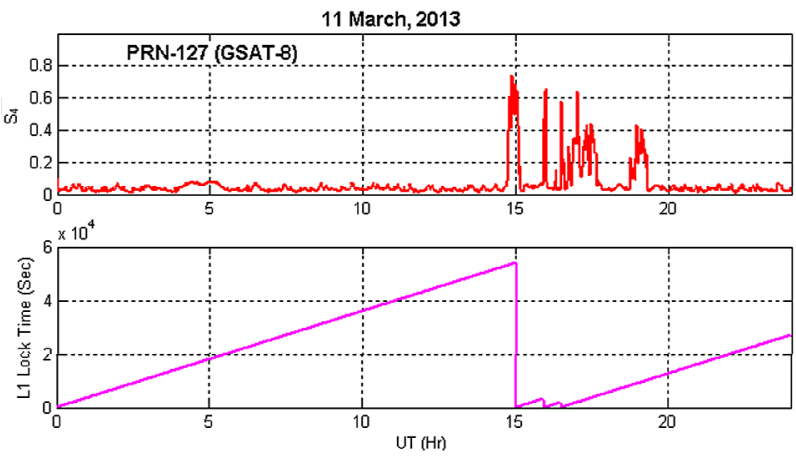

(a)
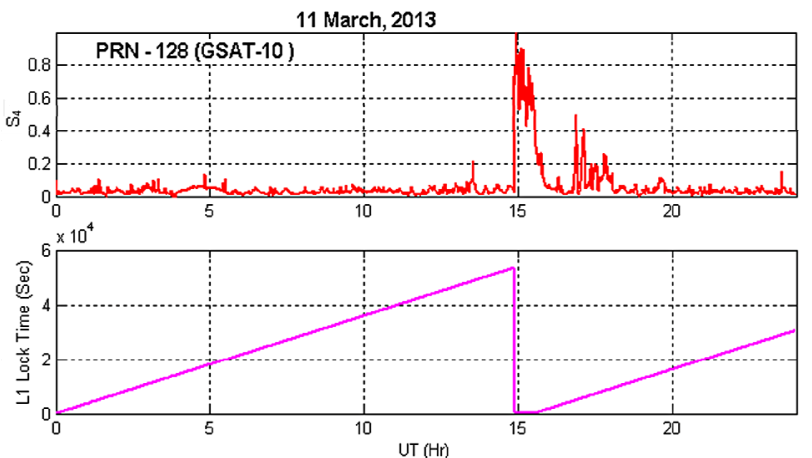

(b)

Figure 6. (a) (upper panel) Amplitude Scintillation $\mathrm{S}_{4}$ observed at GSAT-8 from Ahmedabad on 11 March, 2013 and (bottom panel) corresponding lock time counter (sec.) of L1 SBAS signal; (b) Same as 6(a) but for GSAT-10 located at $83^{\circ} \mathrm{E}$

ionospheric pierce points (IPPs). The loss of lock of SBAS satellite at the user receiver may result in missing the correction message and more importantly, the integrity messages, which are very critical for safety of life applications like civil aviation. Such simultaneous loss of 
lock of GEO satellites has not been reported so far in any other operational SBAS system.

After highlighting the direct effect of loss of lock on SBAS, we will further discuss how the loss of lock of GPS satellites indirectly affects the SBAS service availability. It can be anticipated by now that severe scintillation on 11 March, 2013 might have caused wide-spread loss of lock of the GPS satellites. This results into drop in the number of measurements points (or the IPPs) used for estimation of Grid Ionospheric Vertical Delay (GIVD) at the pre-defined Ionospheric Grid Points (IGPs) by the suitable grid based ionospheric model [2]. In addition to the GIVD, the model also estimates the corresponding error bound known as Grid Ionospheric Vertical Error (GIVE) whose variance is critical for computing the Horizontal and Vertical Protection Limits (HPL/VPL). The drop in the number of measurement points will lead to higher variance of the error. Higher variance, in turn, will lead to higher HPL/VPL which may cross the threshold values of alert limits of APV1.5 navigation service, thus reducing the availability of that particular service.

It is worthy to note that GAGAN uses a unique iono model known as Multi-Layer Data Fusion (MLDF) based on Kriging technique [10]. We have extracted and decoded the ionospheric correction message types 18 and 26 to derive the GIVD and GIVE at the 102 IGPs served by GAGAN. Figure 7(a) shows the GIVE map interpolated over Indian region from the broadcast GIVE values at the IGPs at $14 \mathrm{hr} 56 \mathrm{~min}$ on 11 March, 2013. It clearly indicates the high GIVE values of 15 and $45 \mathrm{~m}$ over the entire GAGAN service area. It may be recalled that we have observed severe scintillation at $\sim 1500$ UT on 11 March, which may have resulted in the increased GIVE values and thus the reduction in service availability. Figure 7(b) provides the reference of GIVE variations during quiet time i.e. at $\sim 0200$ UT exhibiting smaller GIVE values of 3.6 and $4.5 \mathrm{~m}$ over the Indian region.

\subsection{Quantitative Assessment of Loss of Lock}

The direct and indirect effects of loss of lock of the satellites (GPS and SBAS) have been discussed so far. Next, we attempted to investigate the quantitative effect of loss of lock using the GPS data from all the stations of GAGAN-TEC network. To achieve this objective, we selected two typical days-6 and 9 April, 2011. The reason behind selecting these two dates is that the 6 April was severe scintillation day while 9 April was scintillation free day as shown in Figure 8. It can be observed that there are more than 2000 scintillation epochs $\left(\mathrm{S}_{4}>0.4\right)$, the highest ever, on 6 April during the whole month of April 2011. The missing measurements at L1 or L2 or both provide the loss of lock information. Further, the total tracked satellites can be computed as shown in Figures 9(a) and (b) for 6 and 9 April, 2011 respectively at

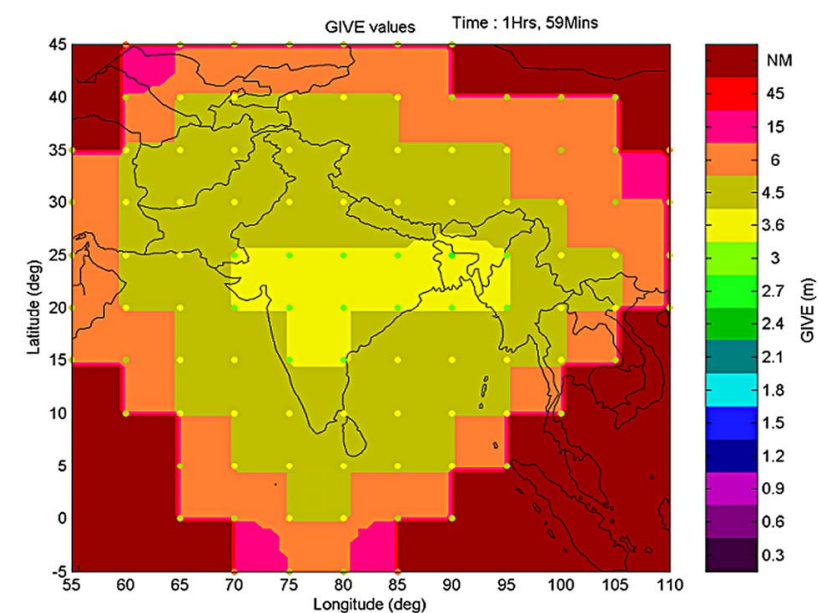

(a)

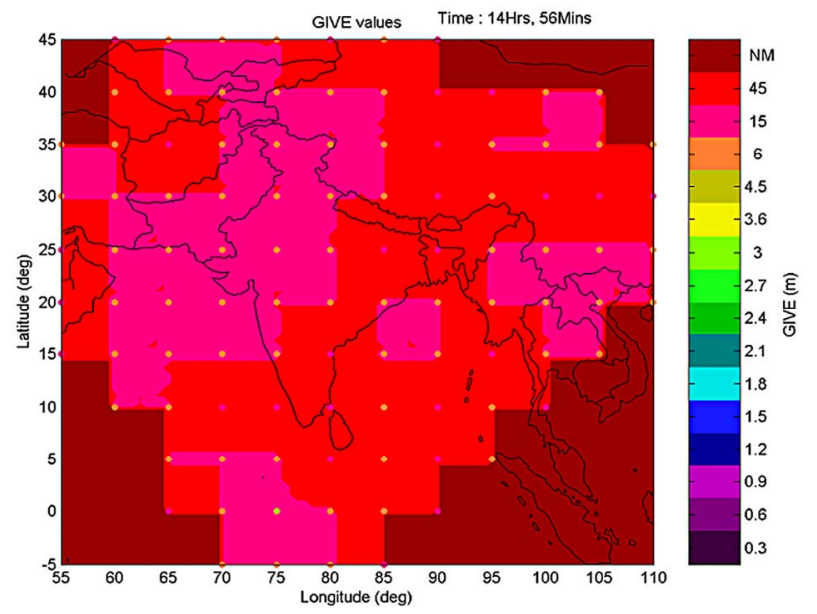

(b)

Figure 7. (a) Grid Ionospheric Vertical Error (GIVE) over the GAGAN service area at 1456 hr (UT) on 11 March, 2013, i.e. during scintillation time. The small dots represent the Ionospheric Grid Points; (b) Same as (a) but during quiet morning time i.e. 0159 hr (UT).

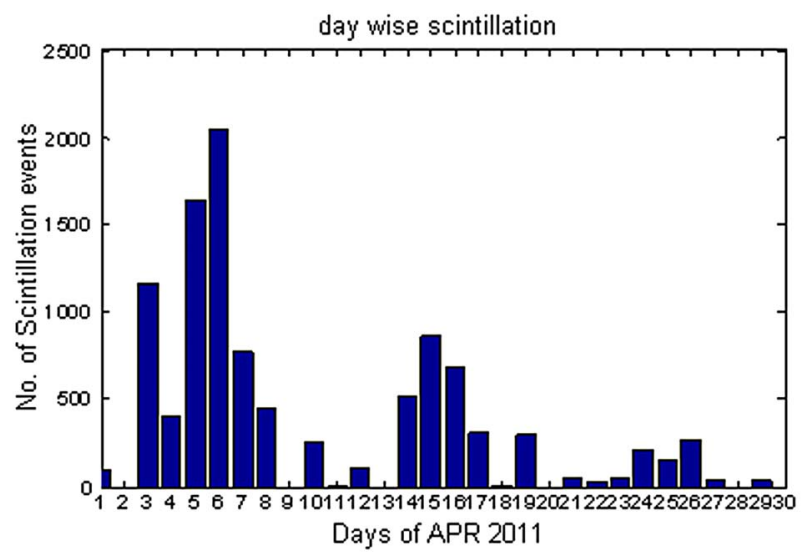

Figure 8. Scintillation statistics during the month of April, 2011 using measurements from all GAGAN-TEC receivers. April 6 recorded highest scintillations while April 9 recorded no scintillation. 

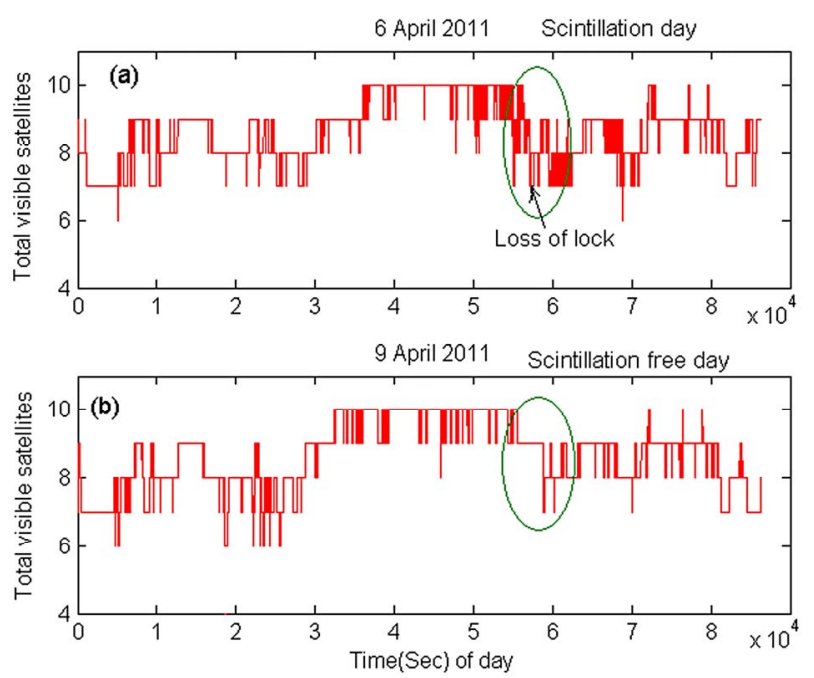

Figure 9. (a) Total number of visible satellites with time at Gaya on 6 April, 2011. (b) Same as (a) but for 9 April, 2011.

Gaya $\left(24.74^{\circ} \mathrm{N}, 84.04^{\circ} \mathrm{E}\right)$. It clearly demonstrates the simultaneous loss of lock in more than 2 satellites as total number of visible satellites drops from 10 to 7 in the circled region of the figure. Such situation may result in poor GDOP thereby reducing the accuracy of position.

The overall impact of scintillation on loss of lock is quantified by calculating the total number of Ionospheric Pierce Points (IPPs), since the number of IPPs will be reduced due to loss of lock. For this purpose, measurements (RINEX data) from all the stations of GAGANTEC network are taken on 6 and 9 April, 2011. The IPP information on 9 April, the scintillation free day, is taken as the reference for comparison. Figure $\mathbf{1 0}$ shows the temporal variation of the total number of IPPs on 6 and 9 April, 2011. The blue markers indicate the IPPs on 9 April, the scintillation free day, while the red markers indicate the same on 6 April, the severe scintillation day. It is clearly evident that the total number of IPPs on 6 April drop down drastically from reference values (April 9), particularly, during scintillation period of $\sim 1400$ to 1900 UT. The maximum impact is observed between 1400 to 1600 UT. It is computed that there is $15 \%$ reduction in the number of IPPs during the severe scintillation period. Such high reduction lead to data under-sampling, resulting in higher error bounds as shown by GIVE map in Figure 7(a).

\section{Summary}

The present paper can be summarised in two sections related to positioning. First is the evaluation of position accuracy using the recently available signal-in-space of GAGAN, an Indian SBAS. The results of the experiment carried out to investigate the position improvement with GAGAN corrections using two collocated dual frequency

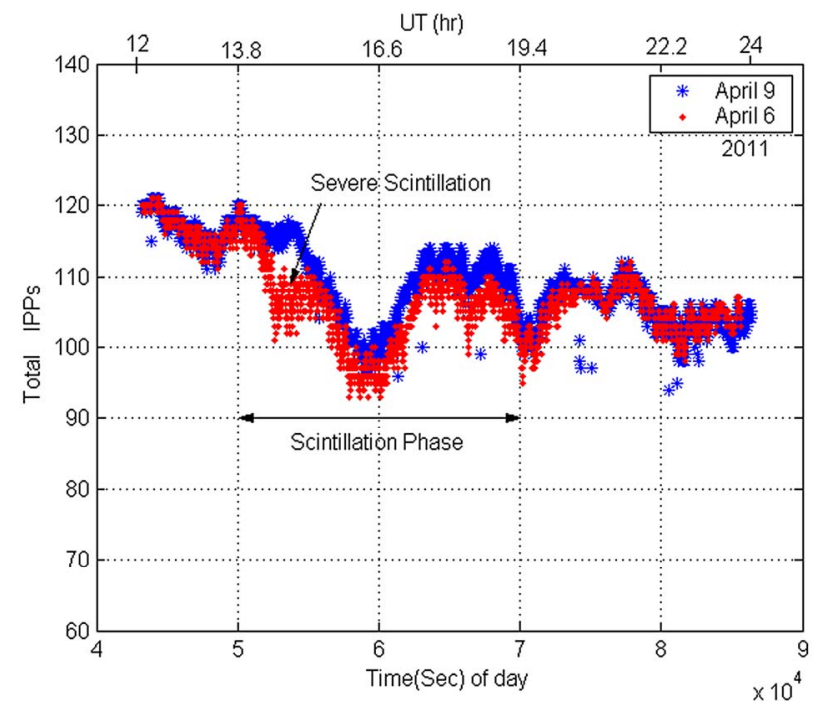

Figure 10. Temporal variation of total number of Ionospheric Pierce Points (IPPs) on 6 April (red) and 9 April, 2011 (blue).

GPS receivers are briefly summarised as:

- Significant improvement in position accuracy is observed by using GAGAN (SBAS) corrections.

- The worst-case error over 2 months of experiment is 5.5 meter in SBAS whereas it is 25 meter in GPS.

- The day-to-day 3-d rms error is better than $1.5 \mathrm{~m}$ in SBAS receiver while the same in GPS stand-alone receiver is more than $3 \mathrm{~m}$.

- The experimental results suggest that GAGAN achieves the required accuracy of $7.6 \mathrm{~m}$ for APV 1.5 navigation service.

Second section deals with the manyfold (direct and indirect) effects of scintillation on GPS as well as on SBAS. We have shown that the position error in GPS as well as in SBAS receiver increases significantly during severe scintillations. The effect of loss of lock due to scintillation was investigated on SBAS and GPS satellites. The results indicate that severe scintillation over a large coverage area can result in loss of lock of both the GEOs, which are widely separated, thus potentially increasing the risk of outage of service and reduced availability. They also affect the grid based iono model by decreasing the number of measurements, thus resulting in higher error bounds. The quantitative assessment of loss of lock over the whole service region indicates $\sim 15 \%$ reduction in the number of measurements (ionospheric pierce points).

\section{Acknowledgements}

We thank the engineers of AAI for operating and maintaining the GPS-TEC receivers at the airports. SS duly acknowledges the support from AAI and SAC, ISRO for providing the facilities in carrying out the research. 


\section{REFERENCES}

[1] R. Acharya, M. R. Sivaraman, K. Bandyopadhyay, N. Nagori, S. Sunda and S. Regar, "Ionospheric Studies for the Implementation of GAGAN," Indian Journal of Radio \& Space Physics, Vol. 36, No. 5, 2007, pp. 394-404.

[2] RTCA SC-159, "Minimum Operational Performance Standard for Global Positioning System/Wide Area Augmentation System Airborne Equipment," RTCA/DO-229B, October 6, 1999.

[3] R. J. Kelly and J. M. Davis, "Required Navigation Performance (RNP) for Precision Approach and Landing with GNSS Application," NAVIGATION: Journal of the Institute of Navigation, Vol. 41, No. 1, 1994, pp. 1-30.

[4] J. Aarons, "Global Morphology of Ionospheric Scintillations,” Proceedings of the IEEE, Vol. 70, No. 4, 1982, pp. 360-378. http://dx.doi.org/10.1109/PROC.1982.12314

[5] S. Basu and K. M. Groves, "Specification and Forecasting of Outages on Satellite Communication and Navigation Systems,” Space Weather, Vol. 125, 2001, pp. 423430. http://dx.doi.org/10.1029/GM125p0423

[6] K. Groves, et al., "Equatorial Scintillation and Systems Support,” Radio Science, Vol. 32, No. 5, 1997, pp. 2047-
2064. http://dx.doi.org/10.1029/97RS00836

[7] P. V. S. Rama Rao, S. Gopi Krishna, K. Niranjan and D. S. V. V. D. Prasad, "Study of Spatial and Temporal Characteristics of L-Band Scintillations over the Indian LowLatitude Region and Their Possible Effects on GPS Navigation," Annales Geophysicae, Vol. 24, 2006, pp. 15671580. http://dx.doi.org/10.5194/angeo-24-1567-2006

[8] P. H. Doherty, T. Dehel, J. Klobuchar, S. H. Delay, S. Datta-Barua, E. R. de-Paula and F. S. Rodrigues, "Ionospheric Effects on Low Latitude Space Based Augmentation Systems," Proceedings of 15th International Technical Meeting (ION GPS), Institution of Navigation, Portland, 2002.

[9] J. Seo, T. Walter and P. Enge, "Availability Impact on GPS Aviation Due to Strong Ionospheric Scintillation," IEEE Transactions on Aerospace and Electronic Systems, Vol. 47, No. 3, 2011, pp. 1963-1973. http://dx.doi.org/10.1109/TAES.2011.5937276

[10] L. Sparks, J. Blanch and N. Pandya, "Estimating Ionospheric Delay Using Kriging: 1. Methodology,” Radio Science, Vol. 46, No. 6, 2011. http://dx.doi.org/10.1029/2011RS004667 